\title{
New chemical sensor for detergents determination
}

\author{
Andrey Bratov, Natalia Abramova, Andrey Ipatov, Angel Merlos \\ Instituto de Microelectronica de Barcelona, Centro Nacional de Microelectronica (CSIC), \\ Campus U.A.B., 08193 Bellaterra, Barcelona, Spain
}

\begin{abstract}
:
A new impedimetric sensor based on an interdigitated electrode array with electrode digits located at the bottom of microcapillaries formed in silicon dioxide is proposed. Microcapillaries are opened at the top, so that in contact with an electrolyte solution the ac current flows close to the surface of the capillary wall from one electrode to another and is significantly affected by changes in surface conductance at the $\mathrm{SiO}_{2} /$ electrolyte interface. Adsorption of detergents on the sensor surface affects the charge distribution in the electrical double layer that is registered as a change in the surface conductance. The sensor may be used to measure a detergent residues starting from $5 \mathrm{ppm}$ even in solutions with high electrolyte conductivity.
\end{abstract}

Key words: Impedimetric sensor, interdigitated electrode array, adsorption of detergents, surface conductance.

\section{Introduction}

In a sustainable society there is a permanent demand of "green" environmentally friendly products among which are typical for any household washing and dish-washing machines. Modern machines use various sensor systems for controlling the washing process to make it energy efficient and water saving. However, there exists a need for sensors that can be used to measure detergents or surfactants residues. Such a sensor could be helpful in reducing the number of rinsing cycles or reducing the amount of water used for rinsing, resulting in both saved water and energy. It also could guarantee safe levels of residue detergent in the washed laundry.

Commonly to control the amount of detergent in washing machines different sensors and systems based on measuring the conductivity of the washing liquid with a detergent are used. Disadvantages of methods based on the conductivity measurement arise from the fact that conductivity of the washing water depends on the concentration of detergent in cases when the final concentration is high. In the rinse cycle, when the concentration of detergent decreases, the conductivity of the solution will depend on the conductivity of the water itself and not the present detergent residues. Therefore, these methods are useful for dispensing and dosing the detergents, but not to control their residues.

\section{Experimental}

Figure 1 schematically presents a new threedimensional interdigitated electrode array. Microcapillaries formed in silicon dioxide over the electrode digits are $3 \mu \mathrm{m}$ wide, $4 \mu \mathrm{m}$ high and opened at the top. Details of the design and technology of sensor fabrication are presented elsewhere [1].

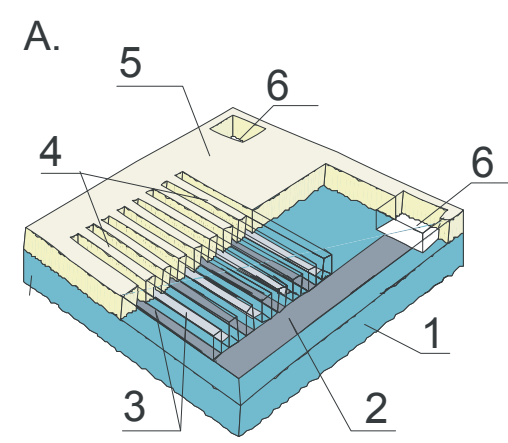

Fig.1. Schematic presentation of the sensor (part of the upper layer is not shown);1 - insulating substrate. 2 - electrode connecting bus, 3 electrodes digits, 4 - capillaries over electrode digits, 5,6 - contact pads.

Characterisation of sensors was performed by impedance measurements in a $100 \mathrm{~Hz}-1000$ $\mathrm{kHz}$ frequency range with a $25 \mathrm{mV}$ (amplitude) voltage excitation using PARSTAT 2263 Advanced Electrochemical System. Z-Plot/ZView software package (Scribner Associates, Southern Pines, NC, USA) was used for 
impedance data treatment and an electrical equivalent circuit fitting.

Measurements were performed in test solutions of various commercial detergents prepared on a tap water with conductivities varying in the range of $300-400 \mu \mathrm{S} / \mathrm{cm}$ at controlled temperature of $25 \pm 0.5^{\circ} \mathrm{C}$ in a thermostatic laboratory environment. At least two identical sensors were tested in the experiments. The conductivity of test solutions was controlled with a commercial conductimeter EC-Meter GLP $31+$ (Crison).

\section{Results and discussion}

As presented in Fig. 2 in an electrolyte solution the ac current applied between the electrodes flows close to the surface of the capillary wall and is significantly affected by changes in surface conductance at the $\mathrm{SiO}_{2} /$ electrolyte interface.

A.

B.
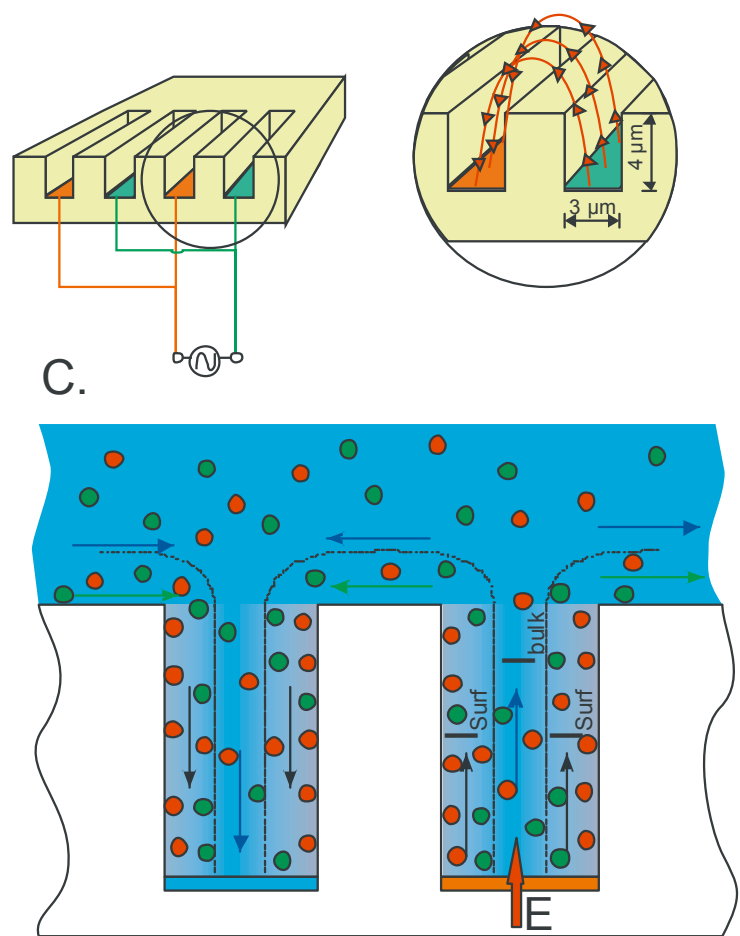

Fig.2. A. Electrical connection of the sensor; $B$. Electric current paths are shown; C. Distribution of ions in microcapillaries and associated bulk and curface currents.

When a tangential electric field is applied at the solid/solution interface, due to a higher concentration of ions within the electrical double layer, the local electric surface current can be significantly different than that of the bulk electrolyte solution (see fig.2 C) especially in diluted electrolyte solutions. Adsorption of charged molecules at the interface alters the charge distribution and thus the surface conductivity. Surfactants that form part of commercial detergents are known to adsorb easily on the silicon dioxide surface [2] affecting the structure and charge distribution in the double layer.

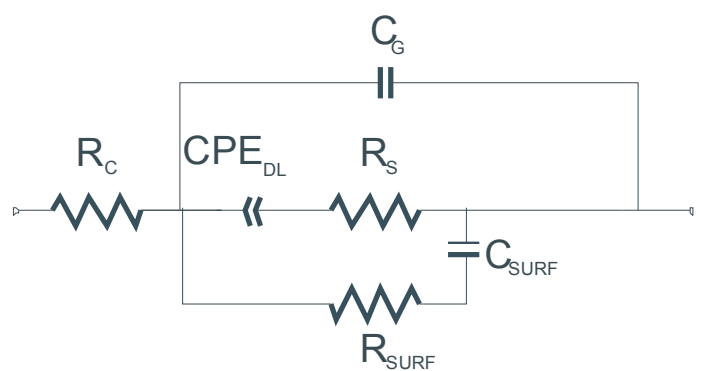

Fig. 3. Equivalent electrical circuit used for spectra fitting.

Analysing the possible electrical equivalent circuit of the microcapillary system presented in fig. $2 \mathrm{C}$ and taking into consideration that the surface conductivity may play an important role, we may distinguish the following components which are presented in figure $3 . R_{C}$ is the contact resistance introduced by wires and collector bars of the electrodes; $C_{G}$ is the geometrical (stray) capacitance between two interdigitated electrodes in a water solution; $R_{S}$ is the resistance of the bulk water solution between two electrodes of the array; $\mathrm{CPE}_{\mathrm{DL}}$ is a constant phase element associated with the capacitance of the electrical double layer at the electrode - water solution interface. The solution bulk resistance, $R_{S}$, is in parallel with the resistance of the surface layer; $R_{\mathrm{SURF}}$, and a capacitive element, $\mathrm{C}_{\text {SURF }}$, between two resistances.

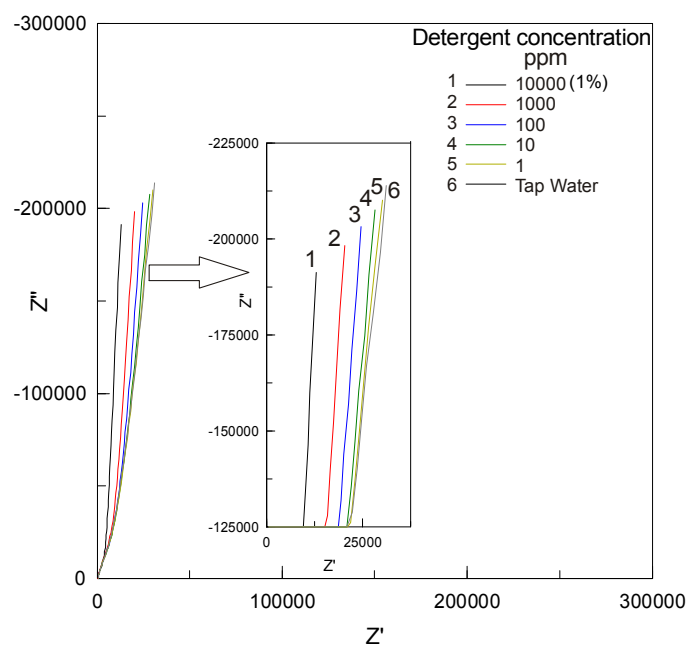

Fig. 4. - Impedance spectra of the sensor measured at different detergent concentrations. ( $Z$ ' and $Z$ ' in Ohms). In the inset low frequency spectra part are shown.

Experimental spectra obtained in solutions with different detergent concentration are presented in fig. 4. As the bulk solution conductivity in 
these experiments remains constant and high $(300-400 \mu \mathrm{S} / \mathrm{cm})$ the high frequency part of the spectra remain unchanged. However, the detergent concentration affects the impedance changes in a low frequency region that may be attributed to changes in a surface concentration of the adsorbed detergent. And indeed, fitting of the spectra with the equivalent circuit presented in fig. 3 showed very good results. The fitting quality was evaluated by chi-squared $\left(x^{2}\right)$ values, which were between $2 \cdot 10^{-4}$ and $9 \cdot 10^{-5}$ in the whole frequency range from $100 \mathrm{~Hz}$ to 1 $\mathrm{MHz}$. The chi-squared value is the square of the standard deviation between the original data and the calculated spectrum and represents an evaluation of the goodness of fit. Performed fitting gave values of $R_{B}$ and $R_{S U R F}$ with relative errors of $0.4 \%$ and $3.5 \%$, respectively. Typical $R_{B}$ and $R_{\text {SURF }}$ values obtained from the spectra were in the range of 300-400 Ohm and 10-50 kOhm, respectively.

It may be noted that $R_{\text {SURF }}$ is the only equivalent circuit element that suffers considerable alterations as the detergent concentration changes. This permits to perform real-time measurements at a fixed frequency of 100-200 $\mathrm{Hz}$ registering the real part of the impedance. Obtained in this way the response curve of the sensor is presented in fig. 5 .

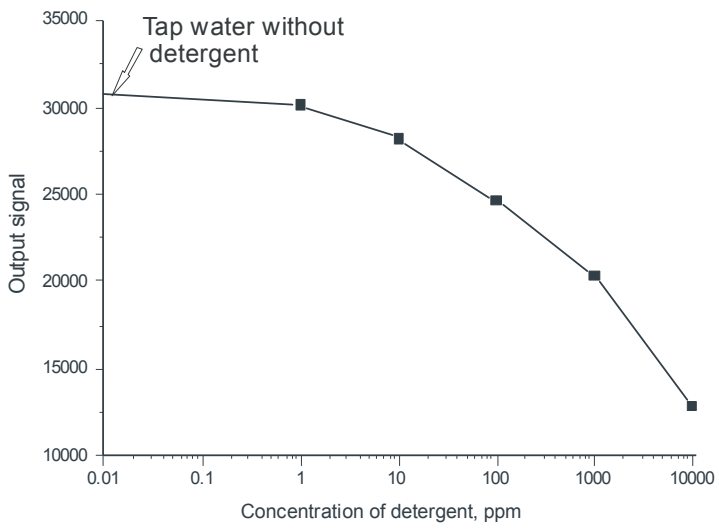

Fig. 5. Response of the sensor to the detergent present in the solution

The detection limit is about $5 \mathrm{ppm}$ of the detergent residue in the solution.

The sensors response time is presented in fig. 6 and is equal to $4 \mathrm{~min}$. After the measurement cycle the sensor, being thoroughly rinsed with the water flow, returns to its initial values and is ready to be used in another measurement cycle.

The sensor response was tested in solutions of different commercial detergents in different aggregate state (powder, gel, liquid) for washing and dish-washing machines as well as in solutions containing technical detergent for cleaning-in-place (CIP) systems. For all the detergents studiedtThe response in the range of concentrations studied $(0-0.1 \mathrm{~g} / \mathrm{l})$ was almost linear with a slope of $-25 \sim-30 \mathrm{k} \Omega$ per $\mathrm{g}$ / I of detergent, which is equivalent to $-25 \sim-30$ $\Omega / \mathrm{ppm}$. The detection limit was $3-5 \mathrm{ppm}$ of detergent.

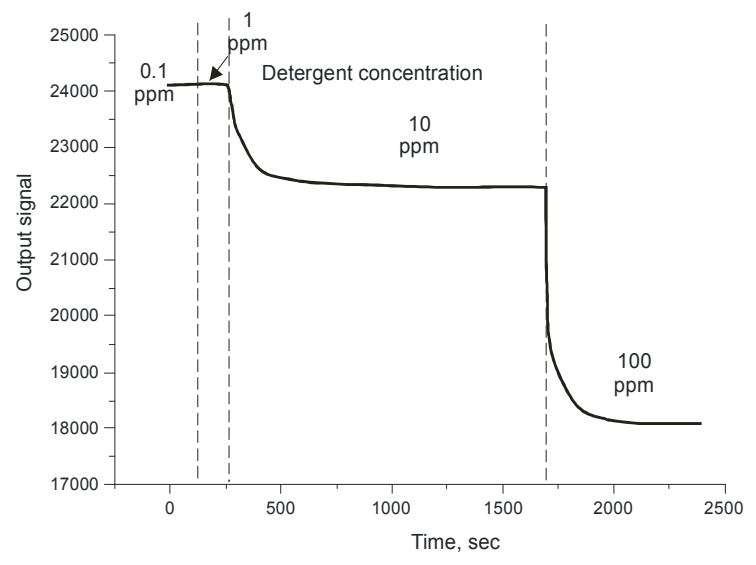

Fig.6. Response time of the sensor to the change in the detergent concentration.

\section{Conclusions}

A new sensor is developed that can be used to control detergents residues in the rinsing cycles. The functional mechanism is based on the measurement of surface conductivity that changes due to the adsorption of surfactants. The sensor was tested in solutions of different commercial household detergents for washing and dishwashing machines. The sensitivity is $-25 \sim-30 \Omega / \mathrm{ppm}$ and the detection limit was 35 ppm of detergent.

The sensor is very robust and simple and could be used in "intelligent" washing machines giving the main processor required information for decisions regarding rinsing, so that the machine could decide if further rinses are necessary. This will result in saved water and energy and guarantee safe levels of residue detergent in the washed laundry, items beneficial for enduser and environment.

\section{Acknowledgements}

Financial support from Spanish Ministry of Science and Innovation (Project AGL200805578-C05-05/01) is greatly acknowledged.

\section{References}

[1] A.Bratov et al. Biosens. Bioelectron. 24 (4), 729735 (2008) 729-735 DOI: 10.1016/j.bios.2008. 06.057

[2] S.Paria, K.C.Khilar, Advances in Colloid and Interface Science 110 (2004) 75-95. DOI: 10.1016/j.cis.2004.03.001 